\title{
ANISOTROPIC FRACTURE CRITERION OF TI6AL4V TITANIUM ALLOY UNDER WIDE RANGE OF STRAIN RATES
}

\author{
Wojciech Moćko, Cezary Kostrzewski \\ Motor Transport Institute, Warsaw, Poland \\ e-mail: wojciech.mocko@its.waw.pl
}

\begin{abstract}
The new fracture criterion taking into account stress triaxiality, strain rate and anisotropy is introduced in this paper. The model is capable to predict the influence of the loading direction on the fracture strain. The equation is applied to estimate the fracture locus of Ti6Al4V titanium alloy under quasi-static and dynamic loading regimes.
\end{abstract}

Keywords: titanium alloys, failure, fracture, anisotropy, Hopkinson bar

\section{Introduction}

In complex structures the fracture behavior depends on the multiaxial stress state which may be defined with the use of the stress triaxiality coefficient. Stress triaxiality is defined as $\eta=-p / q$, where $p$ is the pressure stress and $q$ is the Mises equivalent stress. In the basic approach, the fracture criterion $\bar{\varepsilon}_{f}$ is independent of the stress triaxiality. However, Johnson and Cook (JC) (1985) introduced a new definition of the fracture criterion as a monotonic function of the stress triaxiality in the following form

$$
\bar{\varepsilon}_{f}=\left[C_{1}+C_{2} \exp \left(C_{3} \eta\right)\right]\left(1+C_{4} \ln \frac{\dot{\bar{\varepsilon}}^{p l}}{\dot{\varepsilon}_{0}}\right)\left(1+C_{5} \widehat{T}\right)
$$

where $C_{1}, C_{2}$ and $C_{3}$ are material parameters, $C_{4}$ - strain rate sensitivity, $C_{5}$ - temperature sensitivity, $\dot{\varepsilon}_{0}$ - reference strain rate, $\widehat{T}$ - temperature function described in other papers (Johnson and Cook, 1985).

The JC model given by Eq. (1.1) was applied for the analysis of fracture characteristics of OFHC copper, Armco iron, 7075 T-651 aluminium alloy and 4340 steel (Johnson and Cook, 1985). Further studies of Bao and Wierzbicki (BW) (2005) showed that in the case of some materials, like 2024-T351 aluminium alloy, fracture behavior is govern by two mechanisms, i.e. shear bands and voids formation. The BW fracture criterion taking into account both void formation due to tensile loadings and shear failure may be expressed in the following form (Bao and Wierzbicki, 2005)

$$
\bar{\varepsilon}_{f}=\left\{\begin{array}{lll}
\frac{1}{1+3 \eta} D_{1}+D_{2} & \text { for } & -1 / 3<\eta<0 \\
D_{3} \eta^{2}+D_{4} \eta+D_{5} & \text { for } & 0<\eta<0.4 \\
D_{6} \exp \left(D_{7} \eta\right) & \text { for } \quad 0.4<\eta<0.95
\end{array}\right.
$$

Comparison between the three mentioned fracture criteria is shown in Fig. 1. The curves present JC model calibrated using data obtained for 2024-T351 alloy (Bao and Wierzbicki, 2005) and BW fracture locus estimated for Ti6Al4V alloy (Giglio et al., 2012). It can be observed that the BW criterion reveals two local minimums, the first corresponding to shear loadings (stress 
triaxiality equal to 0) and the second corresponding to pure tensile loadings (stress triaxiality higher than 1). Comparing the JC and BW criteria, it can be stated that for tensile loadings (stress triaxiality higher than 0.4) the fracture strain estimated using both equations gives a comparable predictions, whereas for shear loading conditions the results may be substantially overestimated by the JC fracture model. Depending on the specimen geometry and pre-notch radius, various range of stress traixialities may be obtained (Bao and Wierzbicki, 2004, 2005; Driemeier et al., 2010; Gruben et al., 2011).

(a)

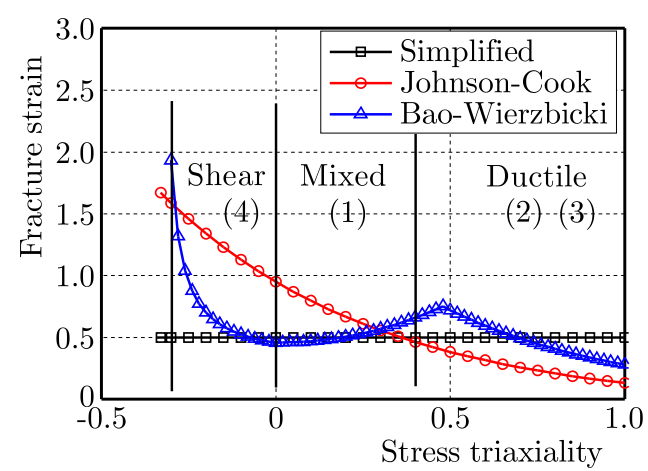

(b)

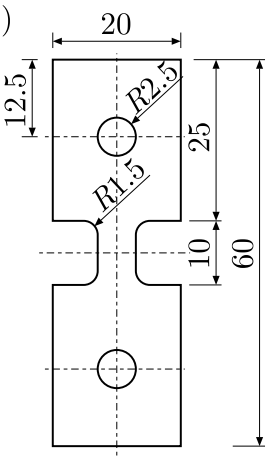

Type A (1)

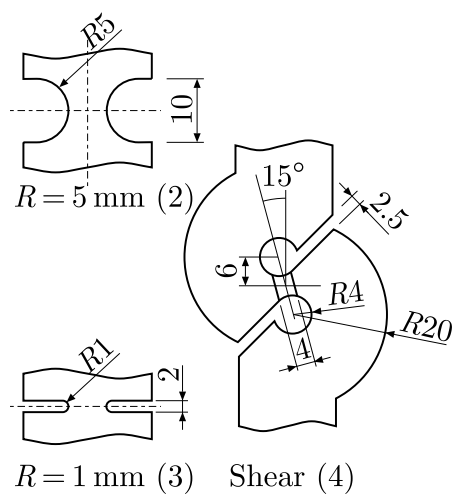

Fig. 1. (a) Comparison of the fracture criterion estimated on the basis of simplified, Johnson and Cook (1985) and Bao-Wierzbicki (2005) theory; (b) specimen geometry and dimensions applied to obtain various stress triaxiality; the number of geometry in figure (b) corresponds to triaxiality marked in figure (a)

Analysis of the ductile fracture locus of Ti6Al4V introduced by Giglio et al. (2012) proved that in the case of this grade of the material, the BW fracture criterion, taking into account both tensile and shear failure, must be considered. The results showed some discrepancies between force-elongation curves obtained experimentally and numerically. One of the probable reasons for these differences may be related to material anisotropy, usually observed for the Ti6Al4V titanium alloy, which was not considered in the cited work (Giglio et al., 2012).

Summarizing, the fracture criterion for majority of ductile materials may be expressed in form proposed by Johnson and Cook or Bao and Wierzbicki. However, in the case of materials with texture introduced by the fabrication process, further studies are required to obtain more accurate fracture models. A new approach should take into account especially the influence of the loading direction on the elasto-plastic and fracture behavior. This works extends earlier analysis (Giglio et al., 2012) of Ti6Al4V fracture criterion by introducing investigation of the titanium alloy anisotropy and deformation strain rate on the fracture locus.

\section{Experimental methodology}

The material was delivered in form of a hot rolled Ti6Al4V titanium alloy sheet of $3 \mathrm{~mm}$ thickness. The specimens were cut along three orientations with respect to the rolling direction, that is, $\mathrm{RD}$ - along, 45D - 45 degree and TD - transverse to the rolling direction. Notched specimens with gauge length equal to $2 \mathrm{~mm}, 5 \mathrm{~mm}$ and $10 \mathrm{~mm}$ were given various stress triaxiality coefficients during tensile test (Fig. 1b). Additionally, shear specimens were designed and fabricated to obtain shear loading conditions (Fig. 1b). The same geometry was applied for both quasi-static and dynamic testing. The specimens were cut using electro-discharge machining (EDM). Tensile tests were carried out at quasi-static and dynamic loading regime using, respectively, with a servo-hydraulic testing machine and split Hopkinson tensile bar (Moćko et al., 2015, 2016). Simultaneously, plastic deformation was recorded and analysed using a digital image correlation 
software to determine strain distribution. Stress triaxiality during tensile tests was calculated using FEM simulation. Analysis was carried out using ABAQUS Standard software under quasistatic loading conditions. Digital models of specimens consists of 24554, 13070, 30555 and 10866 mesh elements, respectively, for type A, R1, R5 and shear samples. For geometry of type A, R1 and R5 C3D8R elements were applied, whereas for shear geometry C3D10M elements were used. Values presented in Fig. 2 are an average value calculated from the mesh elements located near the fracture surface.
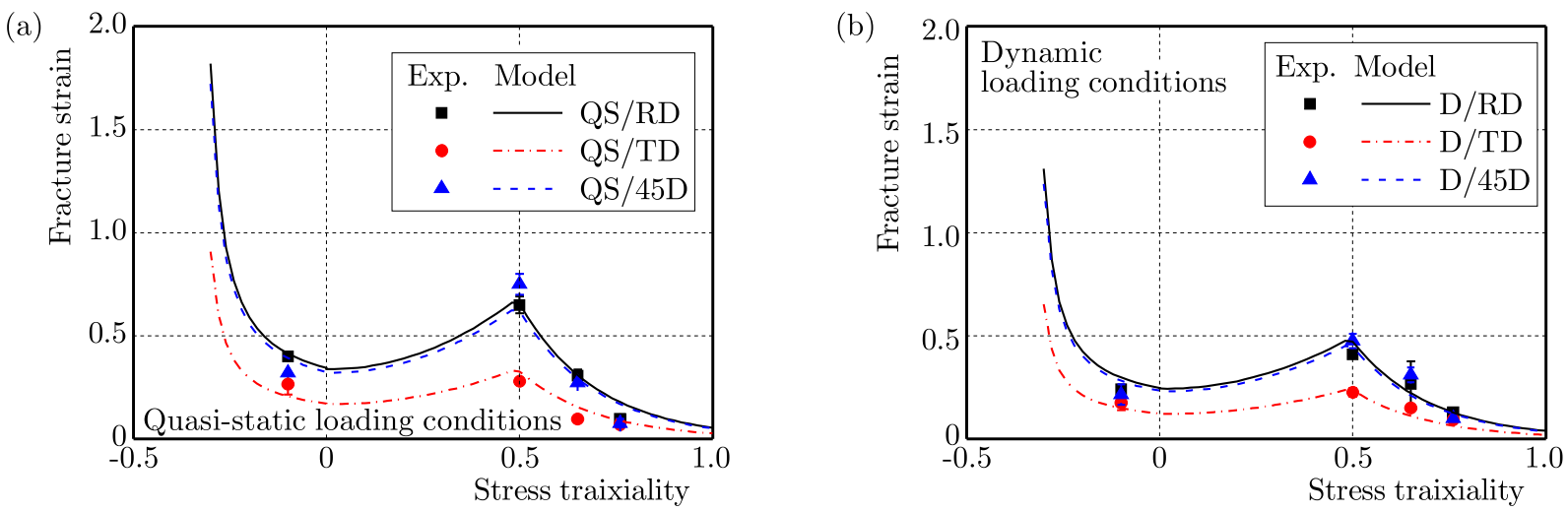

Fig. 2. Comparison of experimental data with predictions of the proposed model at (a) quasi-static and (b) dynamic loading conditions

\section{Results and discussion}

Fracture strain estimated using the digital image correlation method at various stress triaxialities and strain rates is shown in Fig. 2. It can be observed that similarly to the BW model Ti6Al4V titanium alloy loaded at the direction RD and 45D is clear to the observed maximum at the stress triaxiality equal to 0.5 . At stress triaxialities higher than 0.5 , the void formation mechanism is responsible for the fracture, whereas at stress triaxialities lower than 0.5 , the failure of the material is governed by shear band formation or a mixed mechanism. In the case of the loading force transverse (TD) to the rolling direction, the local maximum observed at 0.5 for other orientations is significantly diminished. An other observed phenomenon is the decreasing of the fracture strain with an increase in the strain rate. It may be found that $\mathrm{RD}$ and $45 \mathrm{D}$ orientations are more sensitive to the strain rate effect than TD orientation.

On the basis of experimental results, a new analytical model, including the strain rate and anisotropic effect, has been developed. It is based on the original model proposed by Bao and Wierzbicki (2005) taking into account only the stress state. In the original BW criterion, the fracture strain is expressed as a function of stress triaxiality

$$
\bar{\varepsilon}_{f}=f(\eta)
$$

The experimental results show that in the case of a hot rolled titanium alloy sheet, the fracture model should additionally take into account the effect of strain rate $g\left(\dot{\bar{\varepsilon}}^{p l}\right)$ anisotropy $h(\Theta)$ and temperature $\widehat{T}(T)$ as follows

$$
\bar{\varepsilon}_{f}=f(\eta) g\left(\dot{\bar{\varepsilon}}^{p l}\right) h(\Theta) \widehat{T}(T)
$$

The effect of strain rate may be expressed in form introduced by Johnson and Cook (1985)

$$
g\left(\dot{\bar{\varepsilon}}^{p l}\right)=1+A_{1} \ln \frac{\dot{\bar{\varepsilon}}^{p l}}{\dot{\varepsilon}_{0}}
$$


Anisotropic characteristics of Ti6Al4V titanium alloy are governed by two aspects: firstly, asymmetric geometry of the HCP crystallographic structure and its slipping plane and, secondly, texturing due to cold rolling processing. Results of material anisotropy on the fracture strain may be determined using the following equation

$$
h(\Theta)=1+B_{1} \exp \left(1-\frac{B_{2}}{\Theta}\right) \quad \text { for } \quad \Theta>0
$$

where the loading angle $\Theta$ is estimated as an angle between the rolling direction and the loading force direction.

Finally, the new fracture criterion taking into account stress triaxiality, strain rate and loading direction with respect to rolling directions takes the form

$$
\bar{\varepsilon}_{f}=\left\{\begin{array}{lll}
\left(\frac{1}{1+3 \eta} D_{1}+D_{2}\right) \mathcal{A} & \text { for } & -\frac{1}{3}<\eta<0 \\
\left(D_{3} \eta^{2}+D_{4} \eta+D_{5}\right) \mathcal{A} & \text { for } \quad 0<\eta<0.4 \\
D_{6} \exp \left(D_{7} \eta\right) \mathcal{A} & \text { for } \quad 0.4<\eta<0.95
\end{array}\right.
$$

where

$$
\mathcal{A}=\left(1+C_{1} \ln \frac{\dot{\bar{\varepsilon}}^{p l}}{\dot{\varepsilon}_{0}}\right)\left[1+B_{1} \exp \left(1-\frac{B_{2}}{\Theta}\right)\right]\left(1+C_{2} \widehat{T}\right)
$$

In order to calibrate Eq. (3.5), initially coefficients of the BW fracture criterion $D_{1}-D_{7}$ are estimated using the last square method. Subsequently, the strain rate sensitivity factor $\mathrm{C}$ for the alloy loaded in the rolling direction has been determined. In the final stage of calibration the parameters $B_{1}$ and $B_{2}$ describing anisotropic properties at quasi-static loading conditions have been calculated. Values of the particular coefficients obtained using the mentioned procedure are shown in Table 1. Comparison between the experimental data and predictions of the new model are presented in Fig. 2. The influence of the anisotropy coefficients $B_{1}$ and $B_{2}$ on the fracture strain is shown in Fig. 3. It can be observed that a good agreement between them has been obtained.

Table 1. Coefficients of the new fracture criterion

\begin{tabular}{|c|c|c|c|c|c|c|c|c|c|c|}
\hline$B_{1}$ & $B_{2}$ & $C$ & $\dot{\varepsilon}_{0}[1 / \mathrm{s}]$ & $D_{1}$ & $D_{2}$ & $D_{3}$ & $D_{4}$ & $D_{5}$ & $D_{6}$ & $D_{7}$ \\
\hline \hline 200 & 1.7 & -0.18 & $10^{-4}$ & 0.164 & 0.18 & 1.5 & -0.052 & 0.34 & 8 & -5 \\
\hline
\end{tabular}

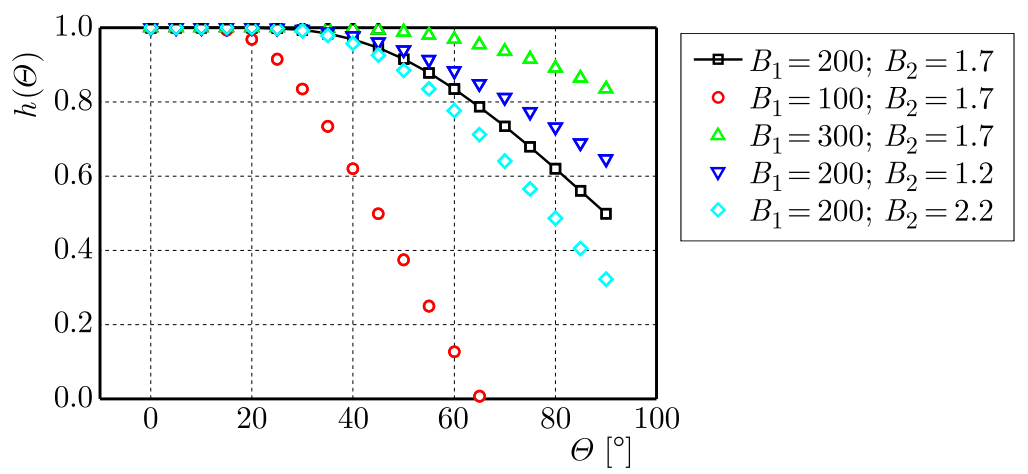

Fig. 3. Influence of the anisotropy coefficients $B_{1}$ and $B_{2}$ on the fracture strain

Acknowledgements

This work was supported by the Motor Transport Institute (grant No. CBM/6506). 


\section{References}

1. Bao Y., WierzBicki T., 2004, On fracture locus in the equivalent strain and stress triaxiality space, International Journal of Mechanical Sciences, 46, 81-98

2. Baо Y., WierzBicki T., 2005, On the cut-off value of negative triaxiality for fracture, Engineering Fracture Mechanics, 72, 1049-1069

3. Driemeier L., Brünig M., Micheli G., Alves M., 2010, Experiments on stress-triaxiality dependence of material behavior of aluminum alloys, Mechanics of Materials, 42, 207-217

4. Giglio M., Manes A., Vigan F., 2012, Ductile fracture locus of Ti-6Al-4V titanium alloy, International Journal of Mechanical Sciences, 54, 121-135

5. Gruben G., Fagerholt E., Hopperstad O.S., Børvik T., 2011, Fracture characteristics of a cold-rolled dual-phase steel, European Journal of Mechanics A/Solids, 30, 204-218

6. Johnson G.R., Cook W.H., 1985, Fracture characteristics of three metals subjected to various strains, strain rates, temperatures and pressures, Engineering Fracture Mechanics, 21, 31-48

7. Moćko W., Brodecki A., Kruszka L., 2016, Mechanical response of dual phase steel at quasistatic and dynamic tensile loadings after initial fatigue loading, Mechanics of Materials, 92, 18-27

8. Moćko W., Brodecki A., Radziejewska J., 2015, Effects of pre-fatigue on the strain localization during tensile tests of DP 500 steel at low and high strain rates, Journal of Strain Analysis for Engineering Design, 50, 571-583 